\title{
Evolutionary Tradeoff and Equilibrium in an Aquatic Predator-Prey System
}

\author{
LAURA E. JONES* AND STEPHEN P. ELLNER \\ Department of Ecology and Evolutionary Biology, \\ Cornell University, \\ Ithaca, NY, \\ USA \\ E-mail: lej4@cornell.edu
}

Due to the conventional distinction between ecological (rapid) and evolutionary (slow) timescales, ecological and population models have typically ignored the effects of evolution. Yet the potential for rapid evolutionary change has been recently established and may be critical to understanding how populations persist in changing environments. In this paper we examine the relationship between ecological and evolutionary dynamics, focusing on a well-studied experimental aquatic predator-prey system (Fussmann et al., 2000, Science, 290, 1358-1360; Shertzer et al., 2002, J. Anim. Ecol., 71, 802-815; Yoshida et al., 2003, Nature, 424, 303-306). Major properties of predator-prey cycles in this system are determined by ongoing evolutionary dynamics in the prey population. Under some conditions, however, the populations tend to apparently stable steady-state densities. These are the subject of the present paper. We examine a previously developed model for the system, to determine how evolution shapes properties of the equilibria, in particular the number and identity of coexisting prey genotypes. We then apply these results to explore how evolutionary dynamics can shape the responses of the system to 'management': externally imposed alterations in conditions. Specifically, we compare the behavior of the system including evolutionary dynamics, with predictions that would be made if the potential for rapid evolutionary change is neglected. Finally, we posit some simple experiments to verify our prediction that evolution can have significant qualitative effects on observed population-level responses to changing conditions.

(C) 2004 Society for Mathematical Biology. Published by Elsevier Ltd. All rights reserved.

\section{INTRODUCTION}

A distinction is often made between ecological and evolutionary timescales, the former referring to relatively rapid changes in the distribution and abundance of species, the latter to more gradual changes in the properties of those species as a result of natural selection. This distinction is tacitly embedded in much of the established theory in ecology and evolution. Ecological models conventionally

\footnotetext{
* Author to whom correspondence should be addressed.

0092-8240/04/061547+27 $\$ 30.00 / 0 \quad$ C 2004 Society for Mathematical Biology. Published by Elsevier Ltd. All rights reserved.
} 
assume constant parameters to characterize species and their interactions-a recent influential text on community ecology (Morin, 1999) does not even list evolution among the 'factors influencing interactions among species'. Conversely, many evolutionary and population-genetic models assume constant population size on the grounds that (relative to the evolutionary time scale) ongoing changes in population size are merely high-frequency 'noise'. When population dynamics are considered, only rare extreme events (bottlenecks, expansion after colonization, etc.) are assumed to matter. Even in theories that explicitly combine ecological and evolutionary dynamics, the separation of timescales is typically assumed. For example, Khibnik and Kondrashov (1997) analyze predator-prey coevolution using singular perturbation theory, assuming that ecological dynamics are much faster than evolution. The 'canonical equation' of Adaptive Dynamics (Marrow et al., 1996; Dieckmann, 1997) used in many recent papers [e.g., Dercole et al. (2003), Le Galliard et al. (2003)] goes even further, and assumes evolutionary changes are so rare that ecological dynamics reach their new asymptotic steady state or attractor before another evolutionary step occurs.

However, recent years have seen an accumulation of evidence that this presumed separation of timescales is often violated [e.g., Thompson (1998), Hairston et al. (1999), Sinervo et al. (2000), Cousyn et al. (2001), Reznick and Ghalambor (2001), Grant and Grant (2002), Yoshida et al. (2003)]: in natural and experimental settings, trait evolution and changes in abundance may occur on similar timescales. For example, Reznick et al. (1997) observed significant life history evolution in guppies over periods of 4-11 years in response to changes in predation pressure, and estimated that evolution occurred in these populations at rates 'up to seven orders of magnitude greater than rates inferred from the paleontological record' (Reznick et al., 1997, p. 1934). Ashley et al. (2003) review evidence for rapid evolutionary changes in response to natural and anthropogenic agents in birds, fishes, mammals, lizards, and plants, with changes occurring in some cases within as little as a single year or generation.

In addition to its theoretical implications, the potential for rapid evolutionary change is critical to understanding how populations adapt to changing environments, for example in harvested or endangered populations where rapid changes in conditions can result from human management or lack thereof (Ashley et al., 2003; Stockwell et al., 2003; Zimmer, 2003). A change in extrinsic factors affecting mortality or reproductive success-such as a change in harvesting rate or juvenile survival-is necessarily a change in the forces of natural selection, and the response is likely to be modified by adaptation. Yet, based on the assumed separation of timescales (and despite evidence that commercial fishing has already led to significant evolutionary changes in harvested species), plans for species management rarely take into account the likelihood that ecological changes will evoke an evolutionary response (Ashley et al., 2003; Stockwell et al., 2003).

In this paper, we continue our studies of the interplay between ecological and evolutionary dynamics, focused on an experimental aquatic predator-prey system 
(Fussmann et al., 2000; Shertzer et al., 2002; Yoshida et al., 2003). Previous studies (summarized below) have shown that major properties of predator-prey oscillations in this system are determined by ongoing evolutionary dynamics in the prey population. Under other conditions, however, the predator and prey tend to apparently stable steady-state densities. These are the subject of the present paper. We study the previously developed model for our system under steady-state conditions, to determine how evolution shapes properties of the steady states. Specifically, we ask:

- Under what conditions do we expect to find single vs. multiple genotypes maintained by selection, and which ones?

- To what extent does evolution affect the responses of the system to changes in external conditions such as resource availability and extrinsic sources of mortality?

The idea that evolution can affect ecological dynamics is certainly not new, indeed a substantial body of theory (reviewed by Abrams, 2000) has developed over several decades to predict how the dynamics and stability of predator-prey interactions can be affected by trait evolution, and how selective harvesting can affect evolution in fish populations (Law and Grey, 1989). However, key assumptions of the models have rarely been verified experimentally, and the practical difficulties of monitoring long-term changes in population abundances and trait distributions in order to test model predictions 'has left a rather unfortunate gap between theory and experiment' (Abrams, 2000, p. 98). 'Although over 40 years of theory have addressed how evolutionary processes can affect the ecology of predator-prey interactions, few empirical data have addressed the same issue' (Johnson and Agrawal, 2003). In the absence of an empirical foundation, there has been a proliferation of speculative models and a corresponding proliferation of alternative predictions: 'Evolution can stabilize or destabilize interactions.... When population cycles exist, adaptation may either increase or decrease the amplitude of those cycles' (Abrams, 2000). In a general model of a 3-species (resource, consumer, predator) food chain, Abrams and Vos (2003) have shown that, as a result of adaptive changes in the consumer, an increase in consumer mortality rate could result in either an increase or a decrease in the resource, consumer (prey), and predator steady-state values.

Our motivation for adding to this literature is to develop specific predictions for an experimentally tractable system, where model assumptions and predictions can be tested with quantitative rigor, allowing direct feedbacks between theory and experiment as in our previous studies with this system (Fussmann et al., 2000; Shertzer et al., 2002; Yoshida et al., 2003). Experimental tests of theoretical predictions are strongest when predictions have been put on record before the experiments are done (Nelson G. Hairston Sr, personal communication). We begin by describing the experimental system and model. We then analyze the model using approaches from evolutionary game theory-that is, 
under a given set of experimental conditions we seek to identify either single ESS prey genotypes (ESS = evolutionarily stable strategy) that cannot be invaded by any alternative genotype, or a noninvasible ESC of coexisting genotypes (ESC $=$ evolutionarily stable combination). We then use the results of this analysis to predict how population response to changing conditions will be modified by adaptive changes in the prey.

The model can produce a wide range of dynamic behavior depending on parameters. A complete analysis of its behavior would be quite lengthy, but also not germane for empirical studies. We therefore consider a wide range of values for parameters that are under experimental control, but for parameters characterizing the organisms we restrict the analysis to values near those estimated to hold in our system. Experimental conditions leading to population cycles require a totally different analysis, which will be the subject of a later paper.

1.1. Experimental system. The experimental system is a predator-prey microcosm with rotifers, Brachionus calyciflorus, and their algal prey, Chlorella vulgaris, cultured together in nitrogen-limited, continuous flow-through chemostat systems. Brachionus in the wild are facultatively sexual, but because sexually produced eggs wash out of the chemostat before offspring hatch, our rotifer cultures have evolved to be entirely parthenogenic (Fussmann et al., 2003). The algae also reproduce asexually (Pickett-Heaps, 1975), so evolutionary change occurs as a result of changes in the relative frequency of different algal clones.

Two parameters of the system can be set experimentally: the concentration of limiting nutrient [nitrate] in the inflowing culture medium $N_{I}$, and the dilution rate $\delta$, the fraction of medium in the chemostat that is replaced each day. A simple ordinary differential equation model given below is able to capture the experimentally observed qualitative behavior of the system: equilibria at low dilution rates, followed by cycling, followed by equilibria and then extinction (Fussmann et al., 2000) as the dilution rate is increased. However, the experimental system exhibited longer-period cycles and unique phase relations which did not match the short-period cycles and classic quarter-cycle predator-prey phase relations predicted by the model. Furthermore, the observed cycles showed extended periods where algal densities were high yet rotifer numbers remained low, followed by rapid growth in the rotifer populations while algal densities remained roughly unchanged.

A series of models were devised to explain these observations (Shertzer et al., 2002), each including some biologically plausible mechanism: rotifer self-limitation via reduced egg fitness when food is scarce; changes in algal nutrient composition as a function of nutrient availability; changes in algal physiology due to accumulation of toxins released by rotifers, and evolved prey defense against rotifer predation. Only the model including prey evolution was able to account well for the qualitative properties of the observed cycles. In that model, algae 
exposed to rotifer predation pressure evolved a 'low palatability' phenotype at some cost to their competitive fitness. Subsequent experiments confirmed the existence of an evolutionary tradeoff between defense against predation and 'fitness' (growth rate in absence of rotifers): 'grazed' algae grown under constant rotifer predation pressure were smaller, competitively inferior, and constituted inferior food (i.e., rotifers grew poorly when fed upon these cells) relative to 'ungrazed' algae grown in rotifer-free environments (Yoshida et al., submitted). Phenotypic differences between grazed and ungrazed algal lineages were heritable-persisting in subsequent generations grown under common conditions-demonstrating that the algal population evolved in response to grazing pressure. Experiments also verified the prediction that predator-prey cycles would exhibit very different qualitative properties (shorter period, and different phase relations) in the absence of prey evolution (Yoshida et al., 2003).

\section{DESCRIPTION OF THE MODEL}

The model is a system of ordinary differential equations describing the predatorprey and prey evolutionary dynamics in our microcosms. It is essentially the same as the model used by Yoshida et al. (2003), with minor simplifications for the sake of analytic tractability. The possibility for genetic variability and evolution in the algal prey population is modeled by explicitly representing the algal population as a finite set of asexually reproducing clones. Each clone is characterized by its 'food value' to the rotifers, denoted $p$ (for 'palatability'). Food value is defined by its effect on the rotifers: $p$ can be thought of as the conditional probability that an algal cell is digested rather than ejected, once it has been ingested. The model thus consists of three equations for the limiting nutrient and rotifers, plus $q$ equations for a suite of $q$ algal clones. In the following equations, $N$ is nitrogen $(\mu \mathrm{mol} / 1), C_{i}$ represents concentration (per liter) of the $i$ th algal clone, where $i=1,2, \ldots, q$; and $R$ and $B$ are Brachionus, fertile and total population counts in individuals per liter, respectively. Fecund rotifers senesce and stop breeding at a rate $\lambda$; all rotifers are subject to fixed mortality $m$. The parameters $\chi_{c}, \chi_{b}$ are conversions between consumption and recruitment rates (additional model parameters are defined in Table 1).

$$
\begin{aligned}
& \frac{d N}{d t}=\delta\left(N_{I}-N\right)-\sum_{i=1}^{q} F_{C, i}(N) C_{i} \\
& \frac{d C_{i}}{d t}=\chi_{c} F_{C, i}(N) C_{i}-F_{b_{i}}\left(C_{i}\right) B-\delta C_{i} \\
& \frac{d R}{d t}=\chi_{b} \sum_{i=1}^{q} F_{b_{i}}\left(C_{i}\right) R-(\delta+m+\lambda) R
\end{aligned}
$$


Table 1. Model parameters.

\begin{tabular}{|c|c|c|c|}
\hline Parameter & Description & Value & Reference \\
\hline$N_{I}$ & $\begin{array}{l}\text { Concentration of limiting nutrient in } \\
\text { supplied medium }\end{array}$ & $80 \mu \mathrm{mol} \mathrm{N} / 1$ & Set \\
\hline$\delta$ & Chemostat dilution rate & Variable $(d)$ & Set \\
\hline$V$ & Chemostat volume & $0.33 l$ & Set \\
\hline$\chi_{c}$ & Algal conversion efficiency & Variable & Fitted \\
\hline$\chi_{b}$ & Rotifer conversion efficiency & Variable & Fitted \\
\hline$m$ & Rotifer mortality & $0.055 / \mathrm{d}$ & F2000 \\
\hline$\lambda$ & Rotifer senescence rate & $0.4 / \mathrm{d}$ & F2000 \\
\hline$K_{c}$ & Minimum algal half-saturation & $4.3 \mu \mathrm{mol} \mathrm{N} / 1$ & F2000 \\
\hline$K_{b}$ & Rotifer half-saturation & $0.835 \times 10^{9}$ algal cells $/ 1$ & TY \\
\hline$\beta_{c}$ & Maximum algal recruitment rate & $3.3 / \mathrm{d}$ & F2000 \\
\hline$\omega_{c}$ & $\mathrm{~N}$ content in $10^{9}$ algal cells & $20.0 \mu \mathrm{mol}$ & F2000 \\
\hline$\epsilon_{c}$ & Algal assimilation efficiency & 1 & F2000 \\
\hline$G$ & Rotifer maximum consumption rate & $5.0 \times 10^{-4} 1 / \mathrm{d}$ & TY \\
\hline$\alpha_{1}$ & Shape parameter in algal tradeoff & Variable, $\alpha_{1}>0$ & Fitted \\
\hline$\alpha_{2}$ & Scale parameter in algal tradeoff & Variable, $\alpha_{2}>0$ & Fitted \\
\hline
\end{tabular}

Set: adjustable experimental parameter.

F2000: Fussmann et al., 2000.

TY: Takehito Yoshida, unpublished data.

$$
\frac{d B}{d t}=\chi_{b} \sum_{i=1}^{q} F_{b_{i}}\left(C_{i}\right) R-(\delta+m) B
$$

where

$$
F_{c, i}(N)=\frac{\rho_{c} N}{K_{c}\left(p_{i}\right)+N}
$$

and

$$
F_{b_{i}}\left(C_{i}\right)=\frac{G C_{i} p_{i}}{K_{b}+\sum_{i=1}^{q} C_{i} p_{i}}
$$

are functional response describing algal and rotifer consumption rates, respectively, and where $\rho_{c}=\frac{\omega_{c} \beta_{c}}{\epsilon_{c}}$.

Equation (6) is derived from the rotifer clearance rate $\mathbf{G}$ (the volume of water per unit time that an individual filters to obtain food), which in this model is a function of algal food value:

$$
\mathbf{G}=\frac{G}{K_{b}+\sum_{i=1}^{q} C_{i} p_{i}}
$$

That is, algae of lower value result in the rotifers increasing their clearance rate, exactly as if the density of food were lower. An alternate model in which rotifer clearance rate does not respond to food value was also considered, but could not fit the experimental data on population cycles as well. 
(a) $\alpha_{1}<1$

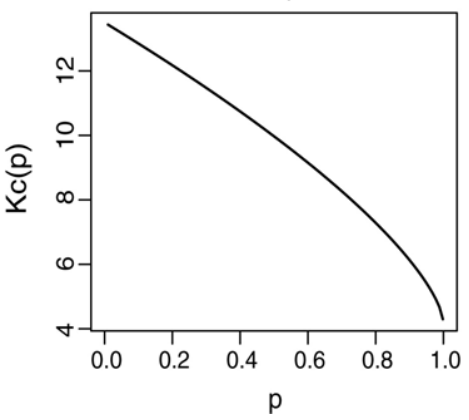

(b) $\alpha_{1}>1$

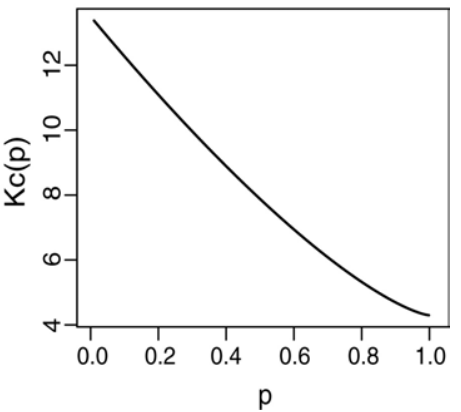

Figure 1. Plots of clonal food value, $p$ vs. half-saturation, $K_{c}(p)$ for shape parameter $\alpha_{1}<1$ [panel (a)] and $\alpha_{1}>1$ [panel (b)]. When the tradeoff curve is concave down (left panel), it is more likely that surviving clones, either in equilibrium or in a cycling regime, represent two extremes in food value. When the curve is concave up (right panel), intermediate algal types can persist.

Each algal clone $c_{i}$ is assigned a food value $p_{i}$ between 1 ('good') and 0 ('bad'), with lower $p_{i}$ resulting in reduced risk of predation. A reduced food value comes, however, at the cost of reduced ability to compete for scarce nutrients. This relationship is specified by a tradeoff curve (Fig. 1), modified from Yoshida et al. (2003). For simplicity, we use a two-parameter family to model how $K_{c}$ varies between $p_{i}=0$ and $p_{i}=1$ :

$$
K_{c}\left(p_{i}\right)=K_{c}+\alpha_{2}\left(1-p_{i}\right)^{\alpha_{1}}
$$

where $K_{c}>0$ is the minimum half-saturation value and $\alpha_{1}, \alpha_{2}>0$ are shape and cost parameters, respectively.

For most parameters we have fairly secure values based on direct experimental measurements (Table 1). For example, the parameters defining rotifer feeding rate were estimated by allowing known numbers of rotifers to graze on algae at known initial densities, with algal density measured again after a fixed amount of feeding time (Yoshida, unpublished). For some parameters, however, we only have indirect estimates obtained by fitting the model to population count data-these parameters are described as 'fitted' in Table 1. Specifically, parameters were chosen to match as closely as possible the amplitude and period of predator and prey cycles, and the phase lag between them, in experimental observations at two different dilution rates where the system exhibits cycles $(\delta=0.69$ and $\delta=0.96)$, and to match the observed lack of cycles at low and high dilution rates. As is often the case, there is a range of parameter values more or less equally consistent with the data, and we explore how this parameter uncertainty affects our predictions. With the best-fitting parameter values, the model [(1)-(6)] produces a bifurcation diagram as a function of dilution rate, $\delta$, which approximates the behavior our system exhibits in the laboratory (Fussmann et al., 2000; Yoshida et al., 2003, supplementary material). 'Low-flow' equilibria exist for dilution rates $\delta \approx 0.05-0.50$, 

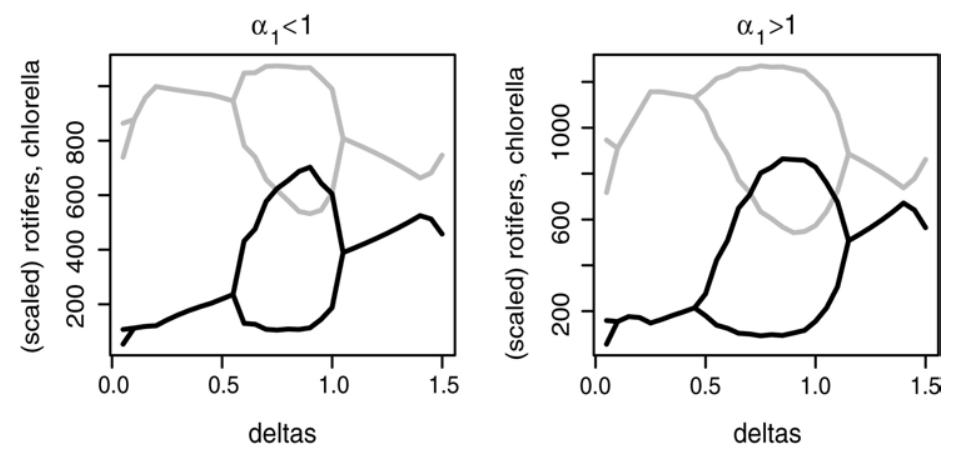

Figure 2. Numerical bifurcation diagrams for the clonal model. Population densities of algae (Chlorella) are shown in gray line; rotifers (Brachionus) in black line. Note the existence of equilibria at both low and high dilution rates. Predator-prey cycles occur at intermediate dilution rates. Parameter sets used in these calculations were obtained by simultaneous fitting of the clonal model [(1)-(6)] to amplitude, phase, and phase-lag observations from an intermediate dilution rate regime (i.e., $\delta=0.69$ ), and a high dilution rate regime $(\delta=0.96)$ during which the system was cycling. Left panel, phase diagram for tradeoff parameter $\alpha_{1}<1: \alpha_{1}=0.78, \alpha_{2}=9.4$, and $p_{\min }=0.10$. Right panel, phase diagram for $\alpha_{1}>1$ : $\alpha_{1}=1.35, \alpha_{2}=9.6$, and $p_{\min }=0.10$.

followed by cycling at intermediate dilution rates, followed by 'high-flow' equilibria at $\delta \approx 1.0-1.5$ (Fig. 2).

\section{Analysis}

This section is the technical part of the paper. For readers who may wish to skim it on first reading, we note that the essential results for subsequent sections are summarized in Figs. 3 and 6.

3.1. Single-clone steady states. Assume now one dominant algal clone $C$ with food value $p$. The model (1)-(4) then reduces to the following four equations, after substituting in the appropriate functional responses (5) and (6):

$$
\begin{aligned}
\frac{d N}{d t} & =\delta\left(N_{I}-N\right)-\frac{\rho_{c} N C}{K_{c}(p)+N} \\
\frac{d C}{d t} & =\chi_{c} \frac{\rho_{c} N C}{K_{c}(p)+N}-\frac{G C p B}{\left(K_{b}+C p\right)}-\delta C \\
\frac{d R}{d t} & =\chi_{b} \frac{G C p R}{K_{b}+C p}-(\delta+m+\lambda) R \\
\frac{d B}{d t} & =\chi_{b} \frac{G C p R}{K_{b}+C p}-(\delta+m) B .
\end{aligned}
$$


(a) Low flow regime

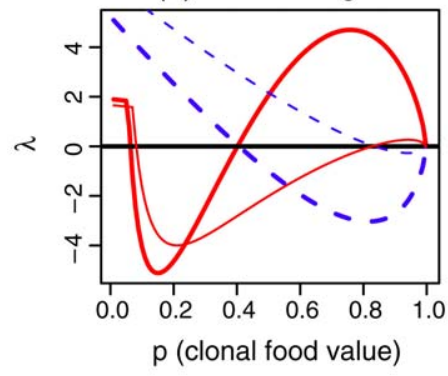

(c) Low flow regime

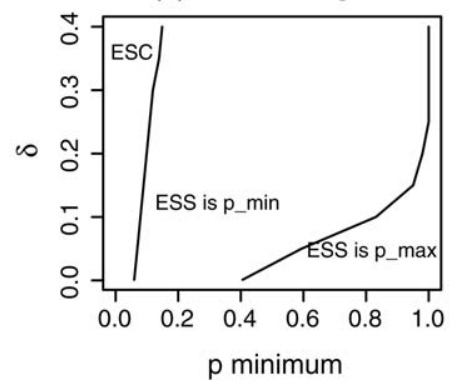

(b) High flow regime

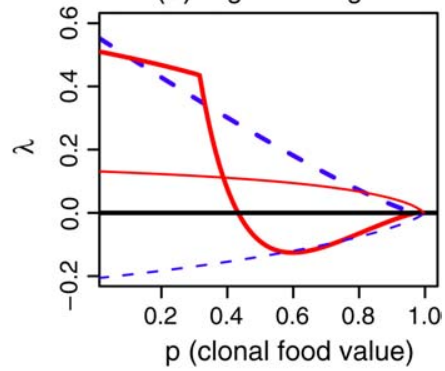

(d) High flow regime

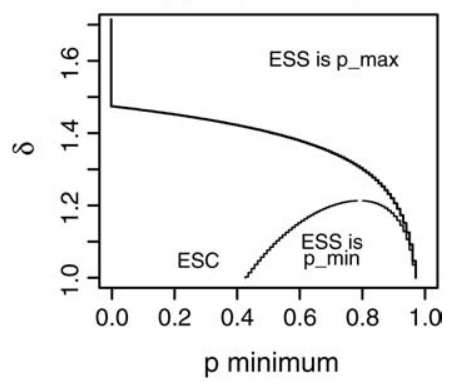

Figure 3. Invasion exponents and evolutionary steady states when $\alpha_{1}<1$. (a) Plots of the invasion exponents $\lambda\left(p_{\max } \mid p\right)$ (solid line) and $\lambda\left(p \mid p_{\max }\right)$ (dashed line) as a function of $p$ for low-flow rates, $\delta=0.001$ (bold) and $\delta=0.15$ (thin). (b) As in panel (a) for high values of $\delta, \delta=1.0$ (bold) and $\delta=1.7$ (thin). Panels (c) and (d) show the predicted evolutionary steady states that result from the plots in (a) and (b) using the criteria in Table 2 , as a function of $\delta$ and $p_{\min }$. The 'corner' in the plot of $\lambda\left(p_{\max } \mid p\right)$ occurs at the minimum food value $p$ that allows persistence of the predators. Above this critical value, $\lambda\left(p_{\max } \mid p\right)$ is calculated for a system steady state including $N, C$, and $B$; below this critical value only $N$ and $C$ are present in the system at steady state.

After some algebra, the steady state for (7) is found to be:

$$
\begin{aligned}
\bar{N} & =\frac{1}{2}\left[-\gamma+\sqrt{\gamma^{2}+4 N_{I} K_{c}(p)}\right] \\
\bar{C} & =\frac{K_{b}(\delta+m+\lambda)}{p\left(\chi_{b} G-(\delta+m+\lambda)\right)} \\
\bar{R} & =\frac{\delta \chi_{b}(\delta+m)\left[\chi_{c}\left(N_{I}-\bar{N}\right)-\bar{C}\right]}{(\delta+m+\lambda)^{2}} \\
\bar{B} & =\frac{\delta \chi_{b}\left[\chi_{c}\left(N_{I}-\bar{N}\right)-\bar{C}\right]}{\delta+m+\lambda}
\end{aligned}
$$

where we define

$$
\gamma=K_{c}(p)-N_{I}+\frac{\rho_{c} \bar{C}}{\delta} .
$$


(a) Low flow regime

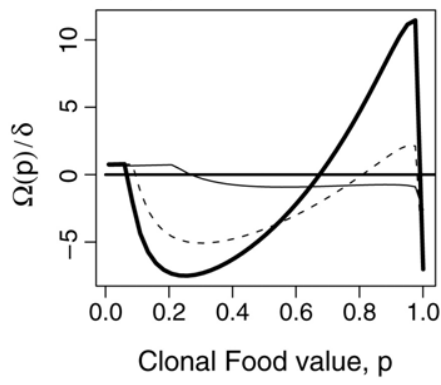

(c) Low flow regime

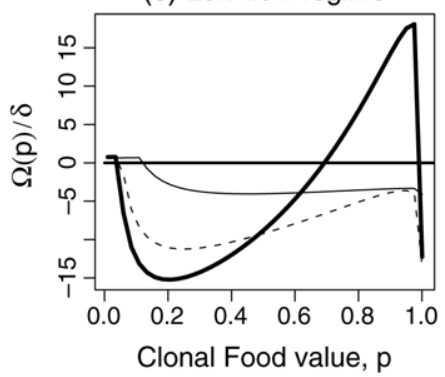

(b) High flow regime

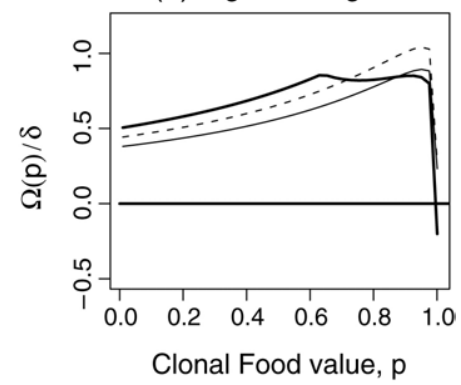

(d) High flow regime

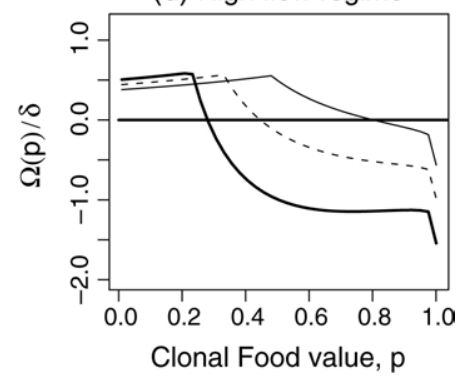

Figure 4. First order conditions (13) for the system when $\alpha_{1}>1$ (note that cost parameter $\alpha_{2}=9.8$ can be classified as 'high' in value, so conditions for limiting dilution rate $\delta \rightarrow 0$ loop above the zero line). (a) $\Omega_{1}$ ( $p$ ) (13), scaled by dilution rate, $\delta$ is shown for the low dilution regime $(\delta=0.1-0.5)$ and conversion efficiency $\chi_{b}=4000$ (thin line). The limiting form for $\delta \rightarrow 0$ is shown in bold line; representative curves for $\delta=0.1$ and $\delta=0.5$ are shown in thin dashed and solid line, respectively. (b) $\Omega_{1}(p)$ for high dilution rates $\delta=1.0-1.5, \chi_{b}=4000$; curve for $\delta=1.0$ is shown in bold; representative curves for $\delta=1.25$ and $\delta=1.5$ are shown in thin dashed and solid line, respectively. (c) As in panel (a) for $\delta=0.1-0.5, \chi_{b}=6000$. Again, the limiting form for $\delta \rightarrow 0$ is shown in bold. (d) As in panel (b) for $\delta=1.0-1.5$ and $\chi_{b}=6000$. Plots for $\delta=1.0$ shown in bold. Note that in the high dilution rate regime, the ESS candidate values move from left to right as dilution rate increases.

Note that these only hold for $\bar{B}>0$. If $p$ is too low or $\delta$ too high, the predators will be unable to persist, and the steady-state nutrient and algal densities are

$$
\bar{N}=\frac{\delta K_{c}(p)}{\left(\chi_{c} \rho_{c}-\delta\right)}, \quad \bar{C}=\chi_{c}\left(N_{I}-\bar{N}\right)
$$

3.2. Conditions for ESS and ESC. We now ask under what conditions a particular clone $C_{r}$, with an associated food value $p_{r}$ might be dominant and noninvasible. Following established usage, a clone $C_{r}$ is said to be an Evolutionarily Stable Strategy [ESS] if a population consisting of $C_{r}$, at steady state, cannot be invaded by a rare alternative clone $C_{i}$ with food value $p_{i}$. The meaning of 'rare' here is that the growth rate of a potential 'invader' $C_{i}$ is computed on the assumption that the 
(a) Low flow regime

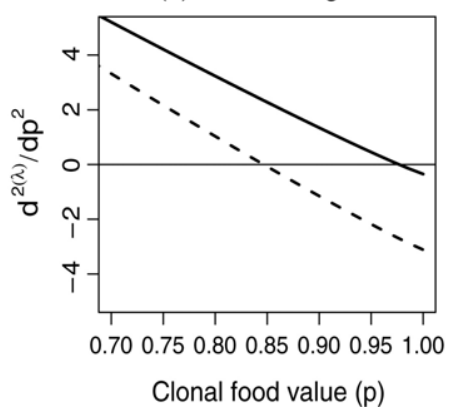

(b) High flow regime

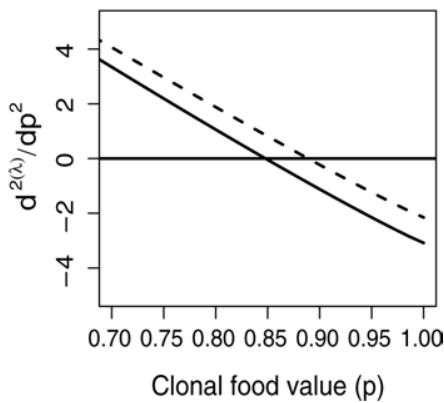

Figure 5. Second order conditions (15) for the system when $\alpha_{1}>1$. Curves represent second order conditions as a function of $p^{*}$ for equilibrium dilution rates $\delta=$ $(0.1-0.5 ; 1.0-1.5)$. (a) Second order conditions for low dilution rate regime $\delta=0.1-0.5$. Dashed lines show curves for $\delta=0.1,0.5$. Heavy bold line shows the limiting form (15) assumes at very low $\delta$ [i.e., $\delta<O\left(10^{-3}\right)$ ]. (b) Second order conditions for the high dilution rate regime $\delta=1.0-1.5$. In bold line is shown the curve for $\delta=1.0$; dashed line shows curve for $\delta=1.5$.

(a) Low flow regime

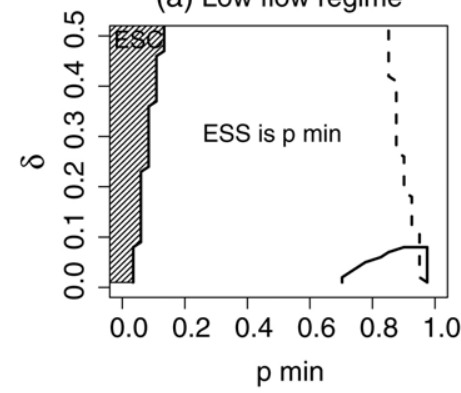

(c) Low flow regime

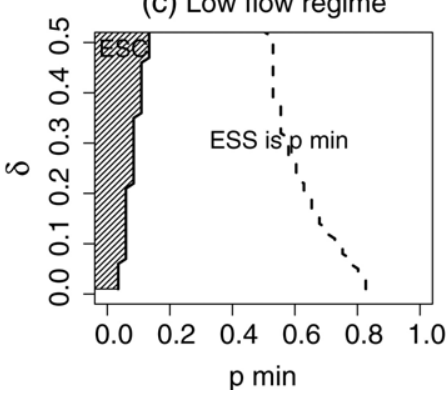

(b) High flow regime

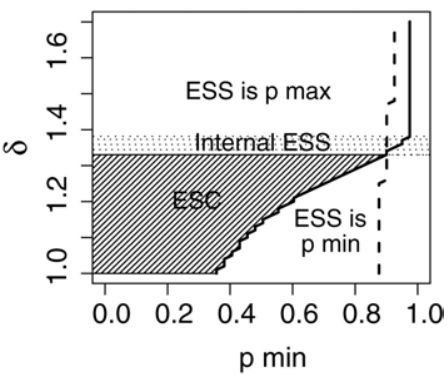

(d) High flow regime

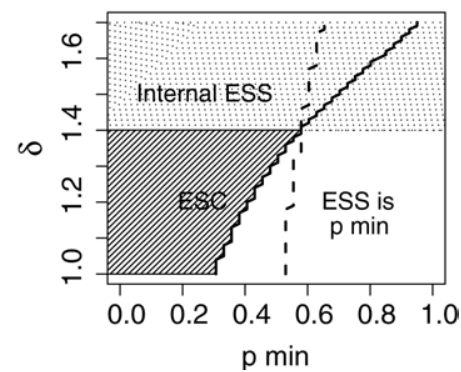

Figure 6. Evolutionary steady states when $\alpha_{1}>1$. ESS candidates are shown in solid line; to the right of the dashed line the second order condition $\partial^{2} \lambda / \partial p_{i}^{2}<0$ is satisfied, so a candidate is an ESS if it lies to the right of the dashed line. If $p_{\min }$ lies to the left of the ESS candidate line, the evolutionarily stable state is either an ESS (if the candidate satisfies the second order condition) or an ESC (if the candidate does not satisfy this condition). Parameters for system shown in panels (a)-(b): $\alpha_{1}=1.08, \alpha_{2}=9.8, \chi_{b}=5420$; panels (c) $-(\mathrm{d}): \alpha_{1}=1.19, \alpha_{2}=3.9, \chi_{b}=6055$. 
population consists entirely of the 'resident' type $C_{r}$. This quantity is now often called the invasion exponent, and is computed as follows:

$$
\lambda\left(p_{i} \mid p_{r}\right)=\frac{1}{C_{i}} \frac{d C_{i}}{d t}=\frac{\chi_{c} \rho_{c} \bar{N}}{K_{c}\left(p_{i}\right)+\bar{N}}-\frac{G p_{i} \bar{B}}{\left(K_{b}+\bar{C}_{r} p_{r}\right)}-\delta
$$

where $\bar{N}, \bar{B}, \bar{C}_{r}$ are equilibrium conditions set by the resident. Note that $\lambda\left(p_{i}\right)$ $\left.p_{i}\right) \equiv 0$ : pitted against itself, a clone neither increases nor decreases.

We can characterize ESSs by the first and second derivatives of the invasion exponent. To be an ESS, an interior trait value $p^{*}$ (i.e., $p_{\min }<p^{*}<p_{\max }$ ) must satisfy both the first order condition, $\partial \lambda / \partial p_{i}=0$ and second order condition, $\partial^{2} \lambda / \partial p_{i}^{2}<0$ at $p_{i}=p_{r}=p^{*}$. A trait value satisfying the first order condition will be called a ESS candidate (Ellner and Hairston, 1994), because the first order condition is necessary but not sufficient. For the first order condition we have

$$
\frac{\partial \lambda\left(p_{i} \mid p_{r}\right)}{\partial p_{i}}=\frac{\chi_{c} \rho_{c} \bar{N} \alpha_{1} \alpha_{2}\left(1-p_{i}\right)^{\left(\alpha_{1}-1\right)}}{\left[K_{c}\left(p_{i}\right)+\bar{N}\right]^{2}}-\frac{G \bar{B}}{\left(K_{b}+\bar{C}_{r} p_{r}\right)}
$$

Setting $\bar{B}^{*}=\frac{G \bar{B}}{\chi_{c} \rho_{c}}$, we see that $p^{*}$ is an ESS candidate if

$$
\Omega_{1}\left(p^{*}\right)=\frac{\bar{N} \alpha_{1} \alpha_{2}\left(1-p^{*}\right)^{\left(\alpha_{1}-1\right)}}{\left[K_{c}\left(p^{*}\right)+\bar{N}\right]^{2}}-\frac{\bar{B}^{*}}{\left(K_{b}+\bar{C} p^{*}\right)}=0 .
$$

Taking the derivative of (12) with respect to the invader's trait value, the second order condition is

$$
\frac{\partial^{2} \lambda}{\partial p_{i}^{2}}=\xi_{1}\left\{2 \alpha_{1} \alpha_{2}\left(1-p_{i}\right)^{\alpha_{1}}-\left(\alpha_{1}-1\right)\left[\bar{N}+K_{c}\left(p_{i}\right)\right]\right\}
$$

where

$$
\xi_{1}=\frac{\chi_{c} \rho_{c} \bar{N} \alpha_{1} \alpha_{2}\left(1-p_{i}\right)^{\left(\alpha_{1}-2\right)}}{\left[K_{c}\left(p_{i}\right)+\bar{N}\right]^{3}}>0
$$

provided $0 \leq p_{i}<1$. Thus, to be an ESS, an interior candidate $p^{*}$ must also satisfy

$$
g\left(p^{*}\right)=2 \alpha_{1} \alpha_{2}\left(1-p^{*}\right)^{\alpha_{1}}-\left(\alpha_{1}-1\right)\left[\bar{N}+K_{c}\left(p^{*}\right)\right]<0 .
$$

Where no single ESS exists-in particular when a candidate fails to satisfy the second order condition-it is often possible to identify a noninvasible ESC (Evolutionarily Stable Combination) of coexisting genotypes. An ESC $\kappa$ of coexisting genotypes is characterized by the invasion exponent for an introduced rare type $p_{i}$,

$$
\lambda\left(p_{i} \mid \kappa\right)=\frac{1}{C_{i}} \frac{d C_{i}}{d t}=\frac{\chi_{c} \rho_{c} \bar{N}}{K_{c}\left(p_{i}\right)+\bar{N}}-\frac{G p_{i} \bar{B}}{\left(K_{b}+\bar{C}_{\kappa}\right)}-\delta
$$


Table 2. Predicted evolutionary outcome as a function of the invasion exponents when $\alpha_{1}<1$.

\begin{tabular}{lll}
\hline & $\lambda\left(p_{\max } \mid p_{\min }\right)>0$ & $\lambda\left(p_{\max } \mid p_{\min }\right)<0$ \\
\hline$\lambda\left(p_{\min } \mid p_{\max }\right)>0$ & $\mathrm{ESC} \kappa=\left\{p_{\min }, p_{\max }\right\}$ & $p_{\min }$ is ESS \\
$\lambda\left(p_{\min } \mid p_{\max }\right)<0$ & $p_{\max }$ is ESS & Both are (local) ESS \\
\hline
\end{tabular}

where $\bar{N}, \bar{B}$, are the steady-state nutrient and rotifer densities set by $\kappa$ and $\bar{C}_{\kappa}$ is the 'effective' algal density at the ESC steady state,

$$
\bar{C}_{\kappa}=\sum_{j \in \kappa} p_{j} \bar{C}_{j}
$$

If $\lambda\left(p_{i} \mid \kappa\right)<0$ for all $p_{i} \notin \kappa$ then $\kappa$ is an ESC. A more refined local classification of evolutionary stability is possible, also based on derivatives of the invasion exponent [Fig. 1 of Levin and Muller-Landau (2000) summarizes the various stability concepts and their relationships], but the ESS and ESC concepts are sufficient for studying our model.

3.3. Shape parameter $\alpha_{1}<1$. We observe from (14) that

$$
\frac{\partial^{2} \lambda}{\partial p_{i}^{2}}>0 \quad \text { when } \alpha_{1}<1
$$

Therefore no interior trait $p$ (i.e., $p_{\min }<p<p_{\max }$ ) can be an ESS if $\alpha_{1}<1$. In addition there cannot be an ESC that includes any interior types (see the Appendix), so any ESC must consist of the extreme types $\left\{p_{\min }, p_{\max }\right\}$. The evolutionary outcome can therefore be determined from the two invasion exponents $\lambda\left(p_{\min } \mid p_{\max }\right)$ and $\lambda\left(p_{\max } \mid p_{\min }\right)$ (Table 2$)$.

Recall that food value $p$ is scaled so that $p_{\max } \equiv 1$, representing the undefended clone that is favored when predators are absent. However, the evolutionary outcome may depend on the value of $p_{\min }$, the food value corresponding to the highest possible level of defense. We consider below a wide range of possible values for $p_{\text {min }}$, i.e., we regard it as being under experimental control rather than a fixed property of the organism. Although the latter is literally true, $p_{\min }$ can be increased temporarily by using a restricted set of founding genotypes as in Yoshida et al. (2003).

Fig. 3 shows plots of the invasion exponents $\lambda\left(p_{\max } \mid p\right)$ and $\lambda\left(p \mid p_{\max }\right)$ [panels (a) and (b)], and the predicted outcome according to the criteria in Table 2 [panels (c) and (d)]. In the low-flow regime [panel (a)], when $p$ is near 0 both $\lambda\left(p_{\max } \mid p\right)$ and $\lambda\left(p \mid p_{\max }\right)$ are positive. Thus, when $p_{\min }$ is in the range of $p$ values where these inequalities hold, we predict an ESC. As $p$ increases, $\lambda\left(p \mid p_{\max }\right)$ remains positive (dashed line) but $\lambda\left(p_{\max } \mid p\right)$ becomes negative (solid line). For $p_{\min }$ in this range of $p$ values, we therefore have $\lambda\left(p_{\min } \mid p_{\max }\right)>0$ and $\left(p_{\max } \mid p_{\min }\right)<0$, 
and the ESS is the minimum existing $p$ (that is, the most defended clone). As $p$ increases further the two exponents reverse in sign, so if $p_{\min }$ lies to the right of this change-point $p_{\max }$ is an ESS.

The high-flow regime is shown in Fig. 3(b). For $p$ very near 1 the situation is the same as at low flow: $\lambda\left(p_{\max } \mid p\right)>0$ and $\lambda\left(p \mid p_{\max }\right)<0$, so we again have $p_{\max }$ as the ESS (these signs follow from the limiting slopes of the curves as $p \rightarrow 1$, derived in the Appendix $)$. As $p$ decreases from $1, \lambda\left(p_{\max } \mid p\right)$ remains positive for higher dilution rates but becomes immediately negative as $p$ decreases from 1 if $\delta \doteq 1$. $\lambda\left(p \mid p_{\max }\right)$ may either remain negative or become positive, depending on the value of $\delta$. When a sign-change occurs, $\lambda\left(p \mid p_{\max }\right)$ is positive and $\lambda\left(p_{\max } \mid\right.$ $p$ ) negative, and we have $p_{\min }$ as an ESS, or both exponents are positive so the predicted outcome is an ESC: a combination of the two extreme types. The location of the sign-change decreases with increasing $\delta$, and eventually vanishes.

The plots shown in Fig. 3 are specific to our parameter set, but we show in the Appendix that the relevant qualitative properties are robust to substantial variation in parameter values.

3.4. Shape parameter $\alpha_{1}>1$ : analysis. The situation is more complicated if $\alpha_{1}>1$, because an internal ESS is then possible. We therefore need to analyze (in this subsection) properties of the first and second order conditions for a local ESS. The implications for evolutionary steady states when $\alpha_{1}>1$ are presented in the next subsection.

First order conditions. We return to the first order expression $\Omega_{1}(p)$ and search for ESS candidate types by exploring where (13) holds as a function of $p^{*}$ and $\delta$, with $0 \leq p^{*}<1$ and $\alpha_{1}>1$. Example plots of $\Omega_{1}(p)$ for different values of the dilution rate $\delta$ and rotifer conversion efficiency $\chi_{b}$ are shown in Fig. 4. Both $\delta$ and $\chi_{b}$ affect the value of $\Omega_{1}(p)$ through the steady-state values $\bar{N}, \bar{B}$, and $\bar{C}^{*}$ (8). Each zero-crossing of an $\Omega_{1}(p)$ curve corresponds to a ESS candidate for that particular parameter set.

For very low dilution rates, $\Omega_{1}(p)$ converges to a limiting form shown in bold [Fig. 4(a) and 4(c); bold line], for which, in this case, there are three ESS candidates. Depending on the value of the cost parameter $\alpha_{2}$, the limiting form may either remain below the $p$ axis (low $\alpha_{2}$ values, one ESS candidate), or loop above it (high $\alpha_{2}$ values, three candidates) as in Fig. 4(a). As dilution rate increases, the $\Omega_{1}(p)$ curve dips entirely below the $p$-axis [Fig. 4(a) and 4(c); thin dashed and solid line], and the two higher- $p$ candidates move together, eventually colliding and vanishing. The trait value(s) of ESS candidates are little affected by variations in rotifer conversion efficiency $\chi_{b}$ in the low dilution regime [Fig. 4(a) and 4(c)].

In the high dilution rate regime, there is a single ESS candidate which increases in $p$-value as dilution rate increases [Fig. 4(b) and 4(d)]. Lower estimates of rotifer conversion efficiency result in higher $p$-value ESS candidates. For example, at $\chi_{b}=4000$, an ESS candidate exists at $p \approx 1$ for $\delta=1.0$ [Fig. 4(b), heavy line]. 
However, at $\chi_{b}=6000$, the candidate for the dilution rate $\delta=1.0$ sits at $p \approx 0.3$ [Fig. 4(d), heavy line]. Higher conversion efficiencies result in greater numbers of rotifers, increased selection for defense, and thus a lower $p$-value ESS candidate.

Most noticeably at higher dilution rates, varying $\alpha_{1}$ also affects the position of the ESS candidate. If $\alpha_{1}$ is increased from some initial value $\alpha_{1}>1$, candidate $p$ values shift to the left. This is sensible when one considers how $\alpha_{1}$ affects the tradeoff curve. Increasing $\alpha_{1}$ makes the curve more deeply concave upwards, while decreasing $\alpha_{1}$ flattens it. As the curve becomes more deeply concave upwards, one might expect surviving clones to migrate in $p$-value towards the middle of the curve, and as it flattens, to move to the extremes - thus, for high $p$-values, to shift towards the high- $p$ endpoint.

Second order conditions. Second order conditions (15) are strongly affected by variation in several parameters, most notably shape parameter $\alpha_{1}$, rotifer conversion efficiency $\chi_{b}$, and dilution rate $\delta$. In the Appendix we demonstrate that $g$ is a decreasing function of both $\alpha_{1}$ and $\chi_{b}$; a decreasing function of $\delta$ in the low dilution range, but an increasing function of $\delta$ in the high dilution range. Consequently, increases in either $\alpha_{1}$ or $\chi_{b}$ shift the second order curve to the left (at a given dilution rate, $\delta$ ), so that lower $p$ value candidates satisfy the second order condition. Conversely, decreasing either $\alpha_{1}$ or $\chi_{b}$ will shift the curve to the right, so that only high $p$ value candidates, can satisfy the second order condition.

The behavior of $g$ with increasing $\delta$ implies that ESS candidates must shift to the right as a function of $\delta$ in order to satisfy the second order condition (15). All else being held constant, for $\alpha_{1}>1$, only relatively high- $p$, high- $\delta$ candidates thus satisfy both the first order (13) and second order conditions. An internal ESS may exist at high $\delta$ equilibria, depending on conversion efficiency $\chi_{b}$, but not at low $\delta$ equilibria.

Plotting (15) as a function of $p$ and $\delta$, we see that low- $\delta$, low- $p$ candidates invariably fail the second order condition, because $g\left(p^{*}\right)$ remains positive for these candidates (Fig. 5). For very low (limiting) $\delta$, there may be high- $p$ candidates which satisfy the second order condition; cf. Fig. 4, panels (a), (c), bold line. The presence or absence of these candidates depends primarily on cost parameter $\alpha_{2}$, as discussed in the next section. However, as $\delta$ increases, the ESS candidate shifts to higher $p$-values, and for these values of $p^{*}, g\left(p^{*}\right)$ becomes negative. Thus while an internal ESS may exist at high dilution equilibria, there is no internal ESS at low dilution rates: a low dilution rate ESS, if it exists, must be an endpoint value.

3.5. Shape parameter $\alpha_{1}>1$ : results. We now use the results above to characterize the evolutionary steady states for $\alpha_{1}>1$. Fig. 6 summarizes the results. Panels (a) through (d) show first and second order conditions for this system together on one plot: solid lines indicate ESS candidates (which we call $p^{*}$ ), and the dashed line is a plot of the second order condition $g\left(p^{*}\right)(15)$. ESS candidates to the left of the dashed line, fail the second order condition; candidates to the right of this 
line satisfy the second order condition. Note that first order conditions for the parameters in Fig. 6(a) and 6(b) are found in Fig. 4(c) and 4(d).

For a low-flow regime with high value of the cost parameter $\alpha_{2}$ and for $p$ small, there are ESS candidates, $p^{*}$ [solid line, panel (a)], but they fail the second order condition. Then, for $p_{\min }<p^{*}$, there is thus an ESC of the two extreme types in proportions such that the ESC has an average $p$ value approximating the ESS candidate value, $p^{*}$. For $p_{\min }>p^{*}$, the first order condition becomes negative and all clones are invasible by $p_{\min }$, which forms an endpoint ESS. For the high costvalue $\left(\alpha_{2} \doteq 10\right)$ example shown in panel (a), there is an additional set of high $p$ ESS candidates at very low dilution rate. However, simulations indicate that these candidates form only a very local ESS and may be invaded by lower $p$ clones. Note that if the cost parameter is lower [e.g., $\alpha_{2}=4$, panel (c)], these high $p$, low $\delta$ ESS candidates do not occur, and only the ESC and low- $p$ endpoint ESS are observed.

In the high-flow regime, ESS candidates $p^{*}$ generally increase in $p$-value with increasing dilution rate [panels (b) and (d), solid line]. For these parameters, however, all ESS candidates for $\delta<1.35$ fail the second order condition. Thus, for $p_{\min }<p^{*}$, there is again an ESC of $p_{\min }$ and $p_{\max }$, in proportions such that the average $p$ value approximates the candidate value $p^{*}$ [panel (b)]. For $p_{\min }>p^{*}$, the first order condition becomes negative, so higher $p$ clones are invasible by $p_{\min }>p^{*}$, and $p_{\min }$ is again an endpoint ESS. However, where ESS candidates do not fail the second order condition, in this case for $\delta>1.35$, an internal ESS exists [above dotted line, panel (b)]. Recall that as $\alpha_{1}$ is increased, we have shown that the second order curve $g\left(p^{*}\right)$ shifts to the left. In this example this causes the intersection between the first and second order curves [dotted line, panel (d)], to occur at lower and lower dilution rates. Thus more and more of the high dilution rate regime has an internal ESS.

Recalling that $\alpha_{1}$ and $\chi_{b}$ affect the first and second order conditions at high dilution rates, we turn to a second example [panels (c), (d)]. Here both $\alpha_{1}$ and $\chi_{b}$ have been increased. Increasing $\alpha_{1}$ shifts ESS candidates to the left, as does increasing $\chi_{b}$. However, this example also features a lower cost parameter $\left(\alpha_{2} \sim 4\right)$, which steepens the first order line such that all ESS candidate types fail the second order condition. Thus as above, for $p_{\min }<p^{*}$, there is an ESC centered about the ESS candidate value, and for $p_{\min }>p^{*}, p_{\min }$ forms an endpoint ESS. Since ESS candidates fail the second order condition for all dilution rates, an internal ESS never exists. The results are otherwise the same as in panel (b).

\section{Simulations}

Because results from the type of ESS analysis we have just completed may only hold locally, we tested our analytical conclusions by performing simulations of the system (1)-(6). The simulations were written in the $\mathbf{R}$ language (version 1.7) and run on a Windows 2000 platform, using the odesolve package. Tests were 
performed for a representative sample of parameter sets with $\alpha_{1}<1$ and $\alpha_{1}>1$ and in a range of dilution rates, as follows.

First, summary diagrams, such as those shown in Fig. 3(c) and 3(d) (for $\alpha_{1}<1$ ) and Fig. 6(a)-6(d) (for $\alpha_{1}>1$ ), were created to show the predicted evolutionary equilibria for each parameter set. Then, each diagram was 'gridded' coarsely in $\left(p_{\min }, \delta\right)$ and simulations of the system in (1)-(6) were run to verify the analysis at each grid point. Some additional 'spot-check' simulations were used to look more closely at predicted transition points, e.g., from ESC to ESS. In each case, the system included an initial set of 40 clones, distributed evenly in $p$ value along the tradeoff curve determined by the parameter set in question. After running a given simulation, we examined time-series trajectories for $\mathrm{C}$, total Chlorella cell counts; average palatability $p$; normalized clonal frequency $c_{i}$ as a function of time; and mean clonal frequency as a function of $p$. Each run verified that (1) the system was in equilibrium ( $p_{\min }$ is a bi-furcation parameter, thus equilibrium must be repeatedly confirmed as $p_{\min }$ varies); and (2) the clone or clones identified by analysis were indeed dominant. In only one case, noted above, did we find that an ESS identified by our analysis was only local in nature, and thus invasible by clones of much lower $p$ value.

\section{Evolution and Response to Changing Conditions}

We can now return to the question posed in the Introduction: how important is an understanding of evolutionary dynamics for making quantitative predictions of how the system will respond to 'management' (an externally imposed alteration in conditions)? If a change in conditions results in a change in community composition, as our analysis and examples suggest, then predictions of how such a community might respond to management must not omit the effects of evolution.

Fig. 7 shows predicted responses of the system to changes in the dilution rate, which affects both nutrient supply and mortality rates. In all panels, the dashed line shows the effects on Chlorella density assuming the system responds to external change by evolving, while the solid line shows the predicted response if effects of evolution are ignored. In panels (a), (b) we assume $\alpha_{1}<1$, and evolutionary steady states are as summarized in Fig. 3(c). In panel (a) we set $p_{\min }=0.75$, then slowly increase the dilution rate from $\delta=0.02$ to $\delta=0.15$, thus crossing from 'ESS is $p_{\max }$ ' to 'ESS is $p_{\min }$ ' on Fig. 3(c). At first both systems respond identically, but at the transition dilution rate ( $\delta \doteq 0.8$ for these parameters), population densities in the evolving system suddenly increase to a higher steady state (dashed line), while those in the nonevolving system follow the original steady state (solid line). In both cases the initial dominant was $p_{\max }$, but only the evolutionarily dynamic system shifted to $p_{\min }$ when conditions changed so that $p_{\min }$ was favored by selection.

In panel (b) we run the converse experiment: we set $p_{\min }=0.75$, but start at dilution rate $\delta=0.15$. We then decrease the dilution rate slowly back down to 
(a)

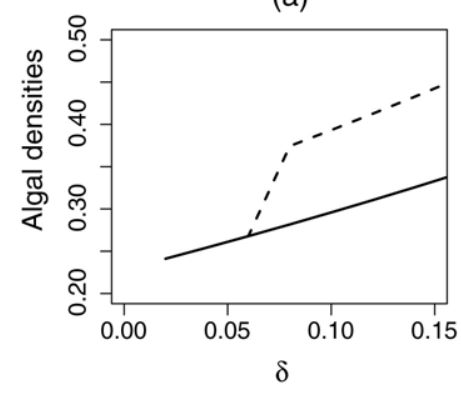

(c)

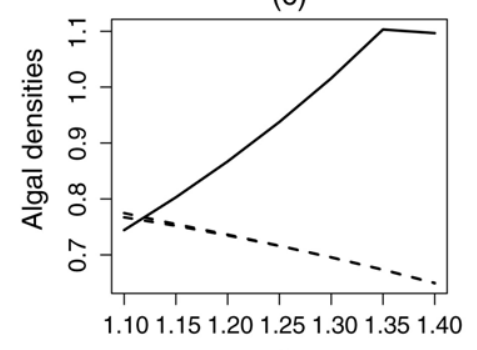

$\delta$

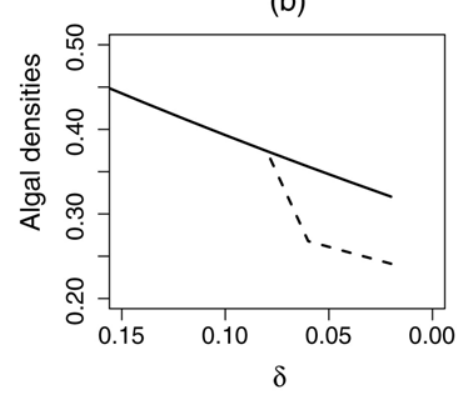

(d)

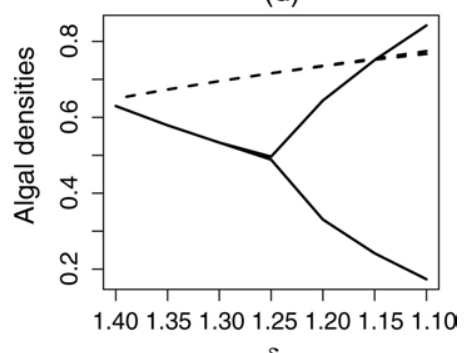

$\delta$

Figure 7. Effects of change in dilution rate on steady-state algal density in an evolutionarily dynamic system vs. a static (nonevolving) system, for $\alpha_{1}<1$ [panels (a), (b)] and $\alpha_{1}>1$ [panels (c), (d)]. Panels (a), (b): $p_{\text {min }} \doteq 0.75$; solid line shows static response, and dashed line shows dynamic response. Parameters and evolutionary dynamics are as in Fig. 3(c). (a) Initial dilution rate $\delta=0.02$, increased to $\delta=0.15$. (b) Initial dilution rate $\delta=$ 0.15 , decreased to $\delta=0.02$. Panels (c), (d): parameters and evolutionary dynamics are as in Fig. 6(d). The dynamic system (dashed line) includes 40 clones; the static system (solid line) is a single-clone whose parameters reflect the aggregate properties of the ESC. (c) Initial dilution rate $\delta=1.1$, increased to $\delta=1.4$. (d) Initial dilution rate $\delta=1.4$, decreased to $\delta=1.1$.

$\delta=0.02$, thus passing back from 'ESS is $p_{\text {min }}$ ' [Fig. 3(c)] to 'ESS is $p_{\max }$ '. Both systems begin with $p_{\min }$ as dominant and their response is identical, but as dilution rate passes through the transition at $\delta \doteq 0.08$, the evolving system switches to $p_{\text {max }}$ as dominant, resulting in lower algal densities (dashed line), the nonevolving system continues to be dominated by $p_{\min }$ (solid line). So up to a point, the system's response to changing conditions can be predicted without regard to the underlying evolutionary dynamics, but then a rapid evolutionary response to a small change in conditions causes a discontinuous response that would not be predicted when evolutionary dynamics are ignored.

With $\alpha_{1}>1$ [Figs. 7(c) and 4(d)] there is an immediate divergence between the actual system response, and predictions that ignore the effect of evolution. In panel (c) we choose an initial dilution rate of $\delta=1.10$, and slowly increase the dilution rate to $\delta=1.4$. At the initial dilution rate, the multiple clone system has an ESC comprised of the extremes, $p_{\min }$ and $p_{\max }$, in proportions such that their average $p$ value approximates the ESS candidate at that dilution rate. Algal densities 
(dashed line) slowly fall as dilution rate increases, due to an increase in average $p$ value of the ESC with increasing dilution rate (higher $\delta$ reduces the rotifer density, and thus reduces the selection for unpalatability). The evolutionarily static system has a single clone, whose parameters reflect the aggregate properties of the ESC (for example, its value of $K_{c}$ was set to the nutrient concentration at which the total uptake rate of the ESC clone mix reached half its maximum value). Here, higher dilution does not evoke the evolutionary response of increased palatability in the prey, so rotifers begin to drop out of the system almost immediately, with a consequent steady increase in algal densities (solid line). By $\delta=1.35$, the rotifers are extinct, and algal densities stabilize.

We then run our imaginary experiment the other way [Fig. 7(d)], starting at $\delta=1.4$ and decreasing down to $\delta=1.1$. The evolving system responds with a slow increase in algal densities: this is because the average palatability of clones comprising the ESC is decreasing with $\delta$, with the expected effect on rotifer abundances. The nonevolving system (a single clone mimicking the aggregate properties of the ESC at $\delta=1.4$ ) maintains constant palatability as conditions change, and again its response is very different (note that the system bifurcates to cycles as we pass back through $\delta \doteq 1.25$ ).

We now propose a set of experimental checks for our analysis that are robust to parameter uncertainties, and in particular to the shape of the tradeoff curve. A general prediction for any plausible parameter values is that the evolutionary steady state at very high dilution rates is a single clone (either $p_{\max }$ or an ESS near $\left.p_{\text {max }}\right)$, which is replaced by an ESC as the dilution rate decreases. As dilution rate decreases further, a Hopf bifurcation occurs and the attractor is then a limit cycle. The period and phase relations of predator and prey cycles depends on which prey clones are present (Yoshida et al., 2003).

We therefore predict that if the dilution rate is quickly dropped into the limitcycle regime, the nature of the cycles will (at least initially) depend on the prior dilution rate. Fig. 8 shows this scenario for both $\alpha_{1}<1$ [panels (a), (b)] and $\alpha_{1}>1$ [panels (c), (d)]. In panel (a) $\left(\alpha_{1}<1\right)$, we start with an initial dilution rate of $\delta=1.15$. The predicted outcome is an ESC of the extreme types, $p_{\min }$ and $p_{\max }$ [Fig. 3(d)]. After equilibrium is established, we abruptly step down the dilution rate to $\delta=0.70$. Given the presence of two very different clones, we predict longer cycles with algal and rotifer cycles exactly out of phase (Yoshida et al., 2003). In panel (b) we start with dilution rate $\delta=1.65$, at which we expect an ESS of $p_{\max }$ [Fig. 3(d)]. When the dilution rate is stepped down to $\delta=0.70$, short cycles with classical predator-prey phase relations result, as one would expect from a single-clone system (Yoshida et al., 2003). Panels (c) and (d) show a corresponding step-down experiment for $\alpha_{1}>1$. In both cases, the nature of the cycles that occur immediately after the step-down reveals the genetic diversity that was present before the step-down, and thus lets us test our predictions about the 'hidden' prey diversity at steady state. Note, however, that prey evolution continues to occur after the step-down [panel (d)], so only 
(a)

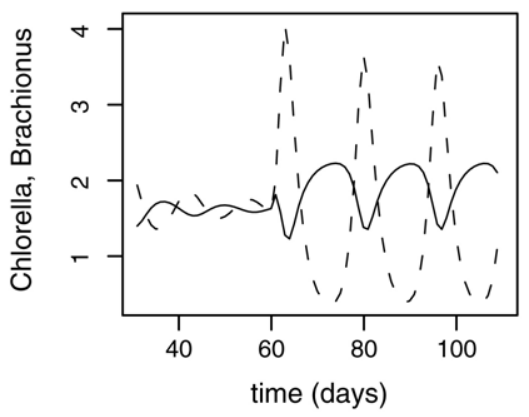

(c)

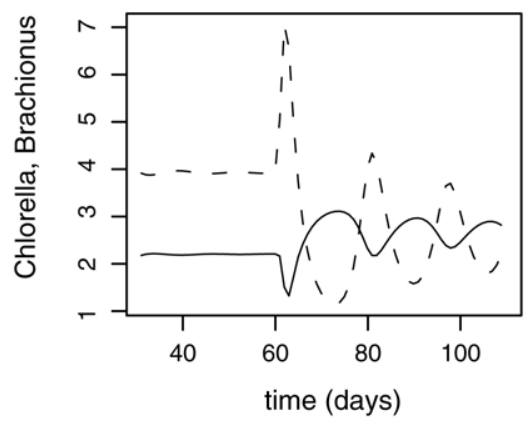

(b)

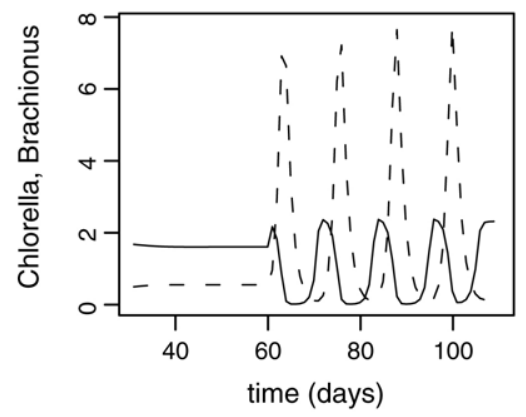

(d)

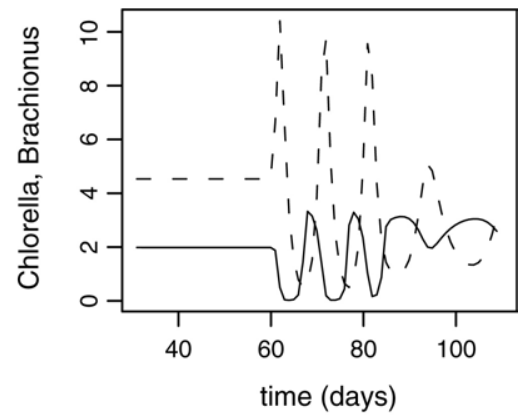

(e)

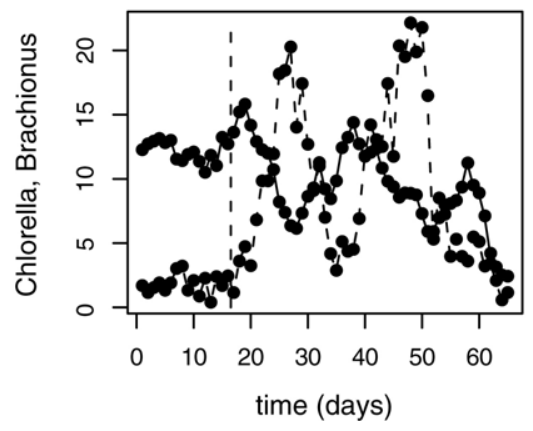

Figure 8. Effects of change in dilution rate on an evolutionarily dynamic system. Rotifer density is shown in dashed line and total Chlorella density in bold line. Panels (a), (b): for $\alpha_{1}<1$. Evolutionary steady states are as in Fig. 3(d); parameters are as follows: $\alpha_{1}=0.765, \alpha_{2}=8.23, p_{\min }=0.07, \chi_{b}=6400$. Panel (a): Initial dilution rate $\delta=1.15$ stepped down to $\delta=0.7$ at $t=60$; (b), initial dilution rate $\delta=1.65$ stepped down to $\delta=0.7$ at $t=60$. Panels (c), (d): for $\alpha_{1}>1$. Evolutionary steady states are as in Fig. 6(b); parameters $\alpha_{1}=1.087, \alpha_{2}=9.79, p_{\min }=0.12, \chi_{b}=5419$. Panel (c), initial dilution rate $\delta=1.2$ stepped down to $\delta=0.7$ at $t=60$; (d), initial dilution rate $\delta=1.45$ stepped down to $\delta=0.7$ at $t=60$. (e), Experimental results (redrawn from Fussmann et al., 2000) corresponding to the simulation experiments in panels (a) and (c); units are $10^{6}$ cells/1 (Chlorella) and cells/1 (Brachionus). At the time indicated by the vertical dashed line, the dilution rate was stepped down from $\delta=1.15$ to $\delta=0.95$. 
the transient behavior after the step-down is revealing of prior evolutionary history.

Panel (e) shows experimental results consistent with our predictions: a step-down experiment from $\delta=1.15$ to $\delta=0.95$ (Fussmann et al., 2000), that was conducted before we were aware of the evolutionary dynamics in the prey population. As predicted for initial dilution rate $\delta=1.15$, the step-down is followed by longperiod cycles having phase relations characteristic of a genetically diverse prey population. What remains to be seen, in future experiments, is whether a stepdown from higher dilution rates would give shorter period cycles with classical predator-prey phase relations, as in panels (b) and (d).

\section{DISCUSSION}

Our analysis of evolutionary dynamics suggests that small, gradual changes in external conditions can precipitate dramatic shifts in community composition or species' densities. This can occur for either qualitative type of tradeoff curve (Fig. 1), through two different mechanisms.

For $\alpha_{1}<1$, extreme prey types are favored and an internal ESS cannot exist. Instead, the prey population is dominated either by an endpoint ESS (the minimum or maximum possible food values), or an ESC consisting of the two extreme types. Under a small change in conditions, the evolutionary steady state can jump from one endpoint ESS to another, resulting in a discontinuous change in species abundances [Fig. 7(a) and 7(b)].

For $\alpha_{1}>1$, an internal ESS may be present at high dilution rates, depending on the shape and cost parameters $\alpha_{1}$ and $\alpha_{2}$, and rotifer conversion efficiency $\chi_{b}$. Where an internal ESS does not exist, the population is dominated either by an endpoint ESS composed of the minimum food value clone present in the system, $p_{\min }$, or an ESC of coexisting types. Simulations indicate that the ESC consists of the two extreme types $p_{\min }, p_{\max }$ in proportions such that the average food value of the population approximates that of the ESS candidate (which satisfies the first order condition but not the second order condition for evolutionary stability). Because the ESC consists of two very different types, the balance between them is strongly affected by relatively small changes in conditions. As a result, the response of the system (including evolutionary changes in the prey) is very different, not only in magnitude but in direction, from what would be predicted if evolutionary changes in the prey are ignored.

Experiments are in progress to determine the shape of the tradeoff curve in our system. Circumstantial evidence supports a tradeoff curve where extreme types are favored $\left(\alpha_{1}<1\right)$ :

- The best fits of the model to qualitative properties of the experimental data (in particular the transition points between stability and cycles as a function of dilution rate, and properties of the cycles) are obtained with $\alpha_{1}<1$. 
- The variability in cycle period (roughly 20-40 days) observed in experiments can occur in the model when $\alpha_{1}<1$ (depending on which clones are present), but not for $\alpha_{1}>1$ (Yoshida et al., 2003).

This raises the possibility that a rapid jump from one extreme prey type to another, as predicted by the model, may be experimentally observable in our system.

Our analysis complements recent work by Abrams and Vos (2003) on a general model for a resource-consumer-predator food chain, with adaptive change in a consumer trait affecting food consumption and mortality rates. Their goal was to predict how perturbations at one trophic level—such as an increase in predator mortality — would propagate through the chain and alter other species' abundances, in the presence of consumer adaptation. The predictions from purely ecological models that ignore consumer adaptation have sometimes been supported, but are also contradicted by numerous experimental studies (Abrams and Vos, 2003). Abrams and Vos (2003) showed that consumer adaptation broadens the range of theoretically possible responses, and so might explain some cases where purely ecological models failed. For example, without adaptation an incremental increase in consumer mortality in their model always entails an increase in resource abundance and a decrease in predator abundance; with consumer adaptation the resource abundance may decrease and predator density may decrease.

Abrams and Vos (2003) consider a spectrum of models with different assumptions about the composition of the consumer trophic level and the nature of response-e.g., assuming a single phenotypically homogenous consumer species responding behaviorally, or a pair of consumer species changing in relative abundance - and found that predictions about total consumer abundance were robust across a range of models. In our analysis the composition of the consumer trophic level is not assumed, but is one of the predicted outcomes from the clonal selection dynamics. As a result, we can predict how prey evolution can lead to qualitative changes in the genetic composition of the prey population, and how these qualitative changes can lead to abrupt population responses to gradual changes in conditions, as well as the gradual changes predicted by the Abrams-Vos models.

Working with well-characterized model ecological systems [e.g., Mueller and Joshi (2000), Cushing et al. (2002)] makes it feasible to study processes that would be far less tractable in the field, and less amenable to rigorous testing of theoretical predictions. Our results lay the groundwork for rigorous tests of the longstanding but still contentious hypothesis that population management must be 'evolutionarily enlightened' (Ashley et al., 2003), rather than continuing to take an exclusively ecological perspective (Stockwell et al., 2003).

\section{ACKNOWLEDGements}

This research was supported by a Mellon Foundation grant to SPE and N G Hairston, Jr. We thank the Cornell EEB eco-theory lunch bunch (in particular 
Parviez Hosseini) and our colleagues on the chemostat project (NGH and Takehito Yoshida) for very helpful comments on our original manuscript that greatly improved its readability. It is conventional to thank the referees for their comments, but in this case it's really true and we are deeply grateful to them for exceptionally careful readings that saved us from errors and ambiguities.

\section{APPENDIX}

$\alpha_{1}<1$. To see that we cannot have an ESC including any interior types, consider a coexisting set $\kappa$ of two or more genotypes, which has an interior member $l$ and some other member $j$. By definition we have $\lambda\left(p_{l} \mid \kappa\right)=\lambda\left(p_{j} \mid \kappa\right)=0$, and as a result of the positive second derivative (18) we conclude that $\kappa$ must be invasible by some other interior genotype near $p_{l}$. Thus, $\kappa$ as defined cannot be an ESC.

We now analyze the qualitative properties of the invasion exponent functions for $\alpha_{1}<1$. Consider first $\lambda\left(p_{\max } \mid p\right)$ for low-flow rate [Fig. 3(a)]. For a resident type with $p$ near 0 we have $\bar{B}=0$ (the predators cannot persist) so defense against predation has no value and any clone with higher $p$ can invade, implying that $\lambda\left(p_{\max } \mid p\right)>0$. As the food value $p$ of the resident type increases, the predators can then persist (this bifurcation accounts for the corner in the plot of $\left.\lambda\left(p_{\max } \mid p\right)\right)$. We can approximate $\lambda\left(p_{\max } \mid p\right)>0$ for $\delta$ small, by inserting the Taylor series expansions of the steady-state values $(\bar{N}, \bar{B}, \bar{C})$ about $\delta=0$ into (11). This gives

$$
\delta^{-1} \lambda\left(p_{\max } \mid p\right)=\frac{1}{p}-\theta_{1}+O(p)+O(\delta)
$$

where $\theta_{1}=N_{I} \chi_{c}\left[\chi_{b} G-(m+\lambda)\right] /\left[K_{b}(m+\lambda)\right]$. The estimated value of $\theta_{1}$ from our experimental data, depending on the value of $\chi_{b}$, is in the range 16.3-26.8, causing $\lambda\left(p_{\max } \mid p\right)$ to become negative roughly at $p=\theta_{1}^{-1} \doteq 0.04-0.06$. Thus, the qualitative behavior of $\lambda\left(p_{\max } \mid p\right)$ at small $p$ is robust to any parameter changes such that $\theta_{1} \gg 1$ continues to hold.

Similarly, for $\delta^{-1} \lambda\left(p \mid p_{\max }\right)$ near $p=0$ we obtain

$$
\delta^{-1} \lambda\left(p \mid p_{\max }\right)=\theta_{1} \frac{K_{c}\left(p_{\max }\right)}{K_{c}(p)}-\left(\theta_{1}-1\right) p-1+O(\delta) .
$$

Thus $\lambda\left(p \mid p_{\max }\right)>0$ for $p$ small if $\frac{K_{c}\left(p_{\max }\right)}{K_{c}(0)}>1 / \theta_{1}$, which is true for our estimated parameters (the LHS is at least $\approx 0.25$, the RHS no more than $\approx 0.06$ ). However, as $p$ increases the $\theta_{2}$ term dominates, and $\lambda\left(p \mid p_{\max }\right)$ becomes negative, as seen in Fig. 3(a). As $\delta \rightarrow 0$ both scaled invasion exponents approach limiting shapes similar to the curve shown in bold [Fig. 3(a)]. 
For low- and high-flow regimes, as $p \rightarrow 1$ the slopes of both invasion exponents become infinite [Fig. 3(a) and 3(b)]. This property results from the value of $\alpha_{1}-$ from (11), with some algebra we obtain that in the limit $p \rightarrow p_{\max }=1$,

$$
\frac{\partial \lambda\left(p_{\max } \mid p\right)}{\partial p} \sim+\text { Constant } \times \frac{\partial K_{c}}{\partial p}
$$

and

$$
\frac{\partial \lambda\left(p \mid p_{\max }\right)}{\partial p} \sim-\text { Constant } \times \frac{\partial K_{c}}{\partial p}
$$

Since $\frac{\partial K_{c}}{\partial p}=-\alpha_{1} \alpha_{2}(1-p)^{\alpha_{1}-1}$ and $\alpha_{1}<1$, then $\frac{\partial \lambda\left(p_{\max } \mid p\right)}{\partial p} \rightarrow-\infty$ and $\frac{\partial \lambda\left(p \mid p_{\max }\right)}{\partial p} \rightarrow+\infty$ as $p \rightarrow p_{\max }$.

$\boldsymbol{\alpha}_{1}>1$. Here we derive the properties of the function $g(p)$ defining the second order condition, that were used in the case $\alpha_{1}>1$. As usual these properties depend on parameter values and our goal is to identify which parameters or parameter combinations control the relevant properties.

(a) $g\left(p^{*}\right)$ is a decreasing function of $\alpha_{1}$ for all $\alpha_{1}>0$

Substituting the steady-state expressions (8) into (15), we rewrite $g$ as a function of $\alpha_{1}$. Taking a partial derivative of this expression with respect to $\alpha_{1}$ yields

$$
\begin{aligned}
\frac{\partial g\left(p^{*}\right)}{\partial \alpha_{1}}= & \frac{K_{c}(p)^{\prime}}{2}\left[\left(3 \alpha_{1}-1\right)-\left(\alpha_{1}-1\right) \frac{\gamma+2 N_{I}}{\sqrt{\gamma^{2}+4 N_{I} K_{c}\left(p^{*}\right)}}\right] \\
& -\left[\bar{N}+K_{c_{\min }}-\alpha_{2}(1-p)^{\alpha_{1}}\right]
\end{aligned}
$$

where $\gamma$ is defined in equation (9), and $K_{c}^{\prime}(p)=\frac{\partial K_{c}(p)}{\partial \alpha_{1}}=\alpha_{2} \ln (1-p)(1-p)^{\alpha_{1}}$.

Note that $K_{c}^{\prime}(p)$ is negative for any $0<p<1$. The expression in the first of the two square brackets is always positive and remains bounded between $\approx 1$.4-2 as $N_{I}$ is either increased from its present value by up to ten times or reduced to zero, and $\chi_{b}$ assumes the range of values we obtained in our optimized parameter sets $\left(\chi_{b}=4000-6500\right)$. The expression in the second square bracket has a lower bound of $\approx 4.5$, and is also always positive. We thus have:

$$
\frac{\partial g\left(p^{*}\right)}{\partial \alpha_{1}} \doteq-[“+"]-[“+”]
$$

and $g\left(p^{*}\right)$ is always a decreasing function of $\alpha_{1}$ for any dilution rate $\delta$ within our experimental range, and for biologically reasonable choices of the experimental parameter $N_{I}$ and the fitted parameter $\chi_{b}$. 


\section{(b) $g\left(p^{*}\right)$ is a decreasing function of $\chi_{b}$}

Substituting the steady-state expressions (8) into (15), we rewrite $g$ as a function of $\chi_{b}$. Taking a partial derivative of this expression with respect to $\chi_{b}$ yields

$$
\frac{\partial g\left(p^{*}\right)}{\partial \chi_{b}}=F(\gamma)=\frac{\left(\alpha_{1}-1\right)}{2} \gamma^{\prime}\left\{1-\frac{\gamma}{\sqrt{\gamma^{2}+4 N_{I} K_{c}\left(p^{*}\right)}}\right\}
$$

where $\gamma$ is defined in equation (9). $F(\gamma)$ thus has the same sign as $\gamma^{\prime}$, which may be written as follows:

$$
\gamma^{\prime}=\frac{\partial \gamma}{\partial \chi_{b}}=-\left\{\frac{\rho_{c} K_{b} G(\delta+m+\lambda)}{\delta p\left[\chi_{b} G-(\delta+m+\lambda)\right]^{2}}\right\} .
$$

The expression within the curly brackets is always positive, thus $F(\gamma)<0$ and thus $g(p)$ is a decreasing function of $\chi_{b}$ for $\alpha_{1}>1$.

(c) $g\left(p^{*}\right)$ is a decreasing function of $\delta$ for $\delta$ small, and an increasing function of $\delta$ when $\delta$ is large for $\alpha_{1}>1$

Again substituting the steady-state expressions (8) into (15), we can rewrite $g$ as a function of $\delta$. Taking a partial derivative of this expression with respect to $\delta$ yields

$$
\frac{\partial g}{\partial \delta}=F(\gamma)
$$

where $F(\gamma)$ is the expression on the right-hand side of equation (A.1) and $\gamma$ is defined in equation (9). As above, $F(\gamma)$ has the same sign as $\gamma^{\prime}$, which may be written as follows:

$$
\gamma^{\prime}=\frac{\partial \gamma}{\partial \delta}=\frac{\rho_{C} K_{b}}{p^{*}}\left\{\frac{(\delta+m+\lambda)^{2}-\chi_{b} G(m+\lambda)}{\left[\delta\left(\chi_{b} G-m-\lambda\right)-\delta^{2}\right]^{2}}\right\} .
$$

This implies that $F(\gamma)<0$ at low $\delta$, and $F(\gamma)>0$ at high $\delta$, switching sign at dilution rate

$$
\delta_{\text {crit }}=-(m+\lambda)+\sqrt{\chi_{b} G(m+\lambda)} .
$$

\section{REFERENCES}

Abrams, P. A. (2000). The evolution of predator-prey interactions: theory and evidence. Annu. Rev. Ecol. Systematics 31, 79-105.

Abrams, P. and M. Vos (2003). Adaptation, density dependence, and the responses of trophic level abundances to mortality. Evol. Ecol. Res. 5, 1113-1132.

Ashley, M. V., M. F. Willson, O. R. W. Pergams, D. J. O'Dowd, S. M. Gende and J. S. Brown (2003). Evolutionarily enlightened management. Biol. Conservation 111, $115-123$. 
Cousyn, C., L. De Meester, J. K. Colbourne, L. Brendonck, D. Verschuren and F. Volckaert (2001). Rapid, local adaptation of zooplankton behavior to changes in predation pressure in the absence of neutral genetic changes. Proc. Natl. Acad. Sci. USA 98, 6256-6260.

Cushing, J. M., R. F. Costantino, B. Dennis, R. A. Desharnais and S. M. Henson (2002). Chaos in Ecology: Experimental Nonlinear Dynamics, Theoretical Ecology Series I, San Diego: Academic Press.

Dercole, F., J. O. Irisson and S. Rinaldi (2003). Bifurcation analysis of a prey-predator coevolution model. SIAM J. Appl. Math. 63, 1378-1391.

Dieckmann, U. (1997). Can adaptive dynamics invade? Trends Ecol. Evol. 12, 128-131.

Ellner, S. P. and N. G. Hairston Jr (1994). Role of overlapping generations in maintaining genetic variation in a fluctuating environment. Am. Nat. 143, 403-417.

Fussmann, G. F., S. P. Ellner and N. G. Hairston Jr (2003). Evolution as a critical component of plankton dynamics. Proc. R. Soc. Lond. B 270, 1015-1022.

Fussmann, G. F., S. P. Ellner, K. W. Shertzer and N. G. Hairston Jr (2000). Crossing the Hopf bifurcation in a live predator-prey system. Science 290, 1358-1360.

Grant, P. R. and B. R. Grant (2002). Unpredictable evolution in a 30-year study of Darwin's finches. Science 296, 707-711.

Hairston, N. G., W. Lampert, C. E. Cceres, C. L. Holtmeier, L. J. Weider, U. Gaedke, J. M. Fischer, J. A. Fox and D. M. Post (1999). Lake ecosystems: rapid evolution revealed by dormant eggs. Nature 401, 446.

Johnson, M. T. J. and A. Agrawal (2003). The ecological play of predator-prey dynamics in an evolutionary theatre. Trends Ecol. Evol. 18, 549-551.

Khibnik, A. I. and A. S. Kondrashov (1997). Three mechanisms of Red Queen dynamics. Proc. R. Soc. Lond. B 264, 1049-1056.

Law, R. and D. R. Grey (1989). Evolution of yields from populations with age-specific cropping. Evol. Ecol. 3, 343-359.

Le Galliard, J. F., R. Ferriere and U. Dieckmann (2003). The adaptive dynamics of altruism in spatially heterogeneous populations. Evolution 57, 1-17.

Levin, S. A. and H. C. Muller-Landau (2000). The evolution of dispersal and seed size in plant communities. Evol. Ecol. Res. 2, 409-435.

Marrow, P., U. Dieckmann and R. Law (1996). Evolutionary dynamics of predator-prey systems: an ecological perspective. J. Math. Biol. 34, 556-578.

Morin, P. (1999). Community Ecology, Oxford: Blackwell Science.

Mueller, L. D. and A. Joshi (2000). Stability in Model Populations, Monographs in Population Biology, 31, Princeton, NJ: Princeton University Press.

Pickett-Heaps, J. D. (1975). Green Algae: Structure, Reproduction and Evolution in Selected Genera, Sunderland, MA: Sinauer Associates.

Reznick, D. N. and C. K. Ghalambor (2001). The population ecology of contemporary adaptations: what empirical studies reveal about the conditions that promote adaptive evolution. Genetica 112-113, 188-198.

Reznick, D. N., F. H. Shaw, F. H. Rodd and R. G. Shaw (1997). Evaluation of the rate of evolution in natural populations of Guppies (Poecilia reticulata). Science 275, 1934-1937.

Shertzer, K. W., S. P. Ellner, G. F. Fussmann and N. G. Hairston Jr (2002). Predator-prey cycles in an aquatic microcosm: testing hypotheses of mechanism. J. Anim. Ecol. 71, 802-815.

Sinervo, B., E. Svensson and T. Comendant (2000). Density cycles and an offspring quantity and quality game driven by natural selection. Nature 406, 985-988. 
Stockwell, C. A., A. P. Hendry and M. T. Kinnison (2003). Contemporary evolution meets conservation biology. Trends Ecol. Evol. 18, 94-101.

Thompson, J. N. (1998). Rapid evolution as an ecological process. Trends Ecol. Evol. 13, 329-332.

Yoshida, T., L. E. Jones, S. P. Ellner, G. F. Fussmann and N. G. Hairston Jr (2003). Rapid evolution drives ecological dynamics in a predator-prey system. Nature 424, 303-306.

Yoshida T., N. G. Hairston Jr and S. P. Ellner, Evolutionary trade off between defense against grazing and competitive ability in a simple unicellular alga, Chlorella vulgaris. Submitted to Proceedings of the Royal Society of London, Biology [PRSLB] (submitted).

Zimmer, C. (2003). Rapid evolution can foil even the best-laid plans. Science 300, 895.

Received 26 November 2003 and accepted 12 February 2004 